\title{
Experiencias en la modalidad de aprendizaje autónomo en línea para la capacitación del funcionariado de la UdelaR
}

\author{
Experiences in the autonomous learning modality in line for the \\ training of civil servants of the UdelaR
}

\author{
Analía Correa \\ Universidad de la República - Uruguay \\ acorrea@cure.edu.uy \\ 0000-0003-2566-7226 \\ Lia Moreira \\ Universidad de la República - Uruguay \\ Imoreirauniversidad@gmail.com \\ (iD) $0000-0001-7511-6479$
}

Fecha de Recepción: 5 de Marzo de 2021

Fecha de Aceptación: 6 de Septiembre de 2021

\section{Resumen}

La sociedad actual, caracterizada por la interconexión, las innovaciones tecnológicas y el flujo de información, requiere de orientaciones institucionales claras que permitan a los individuos desarrollar habilidades para el aprendizaje autónomo en línea. Las Tecnologías de Información y Comunicación se han convertido en instrumentos mediadores y facilitadores del proceso de enseñanza-aprendizaje, ampliando las potencialidades educativas y convirtiéndose en una posibilidad real para la enseñanza formal y no formal. En este sentido, el Instituto de Capacitación y Formación de la Universidad de la República, quien es el encargado de la capacitación y formación del funcionariado técnico, administrativo y de servicios de la Universidad, plantea generar propuestas específicas de capacitación laboral que favorezcan el aprendizaje autónomo en línea, a través del Entorno Virtual de Aprendizaje de la Universidad y de la disposición de herramientas tecnológicas para la comunidad universitaria. El siguiente artículo presenta una relatoría de la experiencia educativa sobre dos cursos ofrecidos por el Instituto en la modalidad de aprendizaje autónomo en línea. Se especifican los contenidos abordados en los cursos, el uso de las diferentes actividades disponibles en el Entorno Virtual de Aprendizaje y de los recursos tecnológicos generados para fomentar el aprendizaje autónomo en línea, los objetos de aprendizaje elaborados en este marco y las propuestas de evaluación de los aprendizajes y de las experiencias, a partir de las percepciones de los participantes. Los aportes de esta relatoría sugieren que la oferta de cursos en esta modalidad de aprendizaje 
autónomo en línea debe ser amplia, diversa, adaptada a las necesidades y demandas de capacitación laboral y sostenida a través del tiempo, para que se pueda mejorar el alcance y gestionar el conocimiento que permita permear la cultura institucional.

Palabras claves: Aprendizaje autónomo en línea, tecnología educativa, capacitación

\section{Abstract}

Today's society, which is characterized by interconnection, technological innovations and the flow of information, requires clear institutional guidelines that allow individuals to develop skills for their autonomous online learning. Information and Communication Technologies have become mediators and facilitators of the teaching/learning process, expanding educational potential and becoming a real possibility for formal and non-formal teaching. In this sense, the Instituto de Capacitación y Formación of the Universidad de la República (UdelaR), which is in charge of training the technical, administrative and service staff of UdelaR, aims at generating specific proposals for job training that favor learning autonomously online through the Entorno Virtual de Aprendizaje of UdelaR and the provision of technological tools for the university's community. The following article presents an educational experience report on two courses offered by the Instituto on the modality of autonomous online learning. The contents addressed in the courses - the use of the different activities available in the Entorno Virtual de Aprendizaje and the technological resources developed to promote autonomous online learning, the learning objects elaborated within the framework of the courses and the proposals are specified and an evaluation of learning and experiences, based on the perceptions of the participants, is made. The contributions of this paper suggest that the courses available in this modality of autonomous online learning should be wide, diverse, adapted to the needs and demands of job training and sustained over time in order to improve the scope and manage knowledge that allows to permeate the institutional culture.

Keywords: Autonomous online learning, educational technology, training

\section{Contexto institucional y protagonistas de la experiencia}

La emergencia sanitaria instalada a nivel global por el avance del SARS-CoV-2 favoreció la multiplicación de propuestas de educación virtual basadas en metodologías autónomas de aprendizaje en línea, debido a que la posibilidad de capacitarse en modalidades más tradicionales se vio ampliamente afectada por la situación sanitaria. A su vez, el trabajo remoto instalado en la Universidad de la República (Udelar), como alternativa para la mitigación de la pandemia, pero en ocasiones en condiciones no óptimas, exigió al Instituto de Capacitación y 
Correa y Moreira - Experiencias en la modalidad de aprendizaje autónomo en línea para la capacita...

Formación (ICF) de la Udelar presentar propuestas de capacitación laboral que permitieran contemplar las diversas realidades del funcionariado.

El aprendizaje autónomo no es una propuesta nueva, de hecho, la educación a distancia siempre ha favorecido este tipo de aprendizajes en línea, la diferencia radica en que, a partir del confinamiento, este tipo de formación pasó a tener un rol preponderante y a ser, en muchos casos, la única o principal opción para mantener los procesos de enseñanza-aprendizaje.

El ICF fue creado por el Consejo Directivo Central (CDC) de la Udelar en agosto de 2018, a través de una ordenanza que plantea que la capacitación es

el conjunto de actividades permanentes, organizadas y sistemáticas, destinadas al desarrollo, perfeccionamiento y actualización de los conocimientos y habilidades necesarios para el desempeño de la función, lo cual contribuirá al logro de los objetivos institucionales, a la mejora de la gestión universitaria y a la optimización de la función pública (CDC, 2018, p. 1).

Este es el organismo central de la Udelar responsable del sistema de capacitación y formación del funcionariado, como parte de las políticas de administración de personal, adoptadas por la institución. Los principios fundamentales de este Instituto son: la formación continua dirigida al funcionariado técnico, administrativo y de servicio con el fin de actualizarlos y/o recalificarlos; la mejora de la gestión universitaria; la certificación de competencias y la mejora de las habilidades y competencias de su funcionariado. El ICF se propone, a través del Plan de Capacitación para el período 2019 a 2023, implementar propuestas de capacitación con metodologías de aprendizaje autónomo en línea, atendiendo no solo a dar una rápida respuesta a las necesidades de capacitación al interior de la organización, sino, además, a propender al desarrollo del capital humano, capaz de actualizar sus conocimientos por sí mismo y cumplir con las exigencias del entorno en permanente renovación. Esto implica, «conjugar una serie de variables que posibiliten el rápido acceso a la información, su adecuada selección, manejo y utilización, pero, además, la habilidad para utilizarla en contextos concretos, en el momento oportuno, con miras a la generación de nuevos conocimientos para la organización» (Argüelles, 2011, p. 150).

En la oferta de cursos del ICF del año 2020, se ofrecieron diversas propuestas de cursos que utilizan esta modalidad o similar en su diseño. Para esta relatoría se seleccionaron dos cursos específicos, cuyo diseño, planificación y evaluación, contemplan los conceptos teóricos que fundamentan el aprendizaje autónomo en línea. Los cursos seleccionados fueron: "Conceptos Básicos sobre Salud y Seguridad» y «Herramientas y aplicaciones de la Web 3.0 para teletrabajar». En el 2020, estos cursos se desarrollaron a través del Entorno Virtual de Aprendizaje 
(EVA) de la Udelar y ofrecieron recursos didácticos como el video informativo, lecturas, foros de comunicación y varias actividades que permitían ejercitar los conceptos adquiridos. Para el curso de «Herramientas y aplicaciones de la Web 3.0 para teletrabajar», se establecieron, además, sesiones en vivo a través de videoconferencia, en las que se orientó a los participantes sobre la metodología de trabajo, las formas de comunicación y modalidades de evaluación.

En los dos cursos se buscó propiciar el aprendizaje autónomo en línea de todo el funcionariado de la Udelar, pero se recomendaban especialmente a aquellos perfiles que pudieran asumir el proceso de enseñanza-aprendizaje autónomamente y tuvieran capacidad para regular sus tiempos y poder cumplir con las actividades establecidas y exigidas para la aprobación de estos.

El curso de "Conceptos Básicos sobre Salud y Seguridad» tuvo una primera edición en 2019 que fue actualizada en 2020 en conocimientos y estrategias didácticas y la propuesta tiene como objetivo que el funcionario vincule conceptos teóricos con aspectos relacionados a su actividad laboral y pueda dar solución a los problemas que surgen en lo cotidiano. Como principal estrategia comunicacional, se dispone del recurso colaborativo del foro que ofrece la plataforma EVA y el seguimiento del tutor.

El curso de «Herramientas y aplicaciones de la Web 3.0 para teletrabajar» se diseñó durante 2020 para generar capacidades que dieran respuesta a las demandas que implicaba la modalidad de teletrabajo instalada a partir del confinamiento. Este curso contó con sesiones en vivo que tenían por objetivo transmitir cómo se iba a desarrollar esta modalidad de aprendizaje y evaluar e intercambiar sobre el proceso de aprendizaje, en cuanto a facilitadores $u$ obstaculizadores que surgieran durante el desarrollo del curso. Los recursos que se dispusieron para este curso constaban de aplicaciones y herramientas informáticas que implicaban que el participante involucrara sus habilidades y conocimientos digitales previos, su experiencia actual y la aplicación de los mismos a su actividad laboral.

En esta experiencia educativa participaron 161 funcionarios de escalafones técnicos, administrativos y de servicios (T/A/S), debido a las temáticas específicas de los cursos, predominaron en el curso de "Conceptos Básicos de Salud y Seguridad» el personal de los escalafones relacionados a Oficios, Servicios Generales, Personal de Laboratorio y Vigilancia, mientras que, en el curso de «Herramientas y aplicaciones de la Web 3.0 para teletrabajar», en su mayoría pertenecían a los escalafones administrativos y técnicos. 


\section{Fundamentos pedagógicos}

La enseñanza apoyada en las Tecnologías de la Información y la Comunicación (TIC) provoca cambios en el proceso de enseñanza-aprendizaje que benefician el compromiso activo del aprendiz, como plantea Salinas,

en el proceso de aprendizaje, la atención a las destrezas emocionales e intelectuales a distintos niveles; la preparación de los jóvenes para asumir responsabilidades en un mundo en rápido y constante cambio; la flexibilidad de los alumnos para entrar en un mundo laboral que demandará formación a lo largo de toda la vida y las competencias necesarias para este proceso de aprendizaje continuo (2004, p. 3).

Este mismo autor plantea que el rol del docente se modifica en un ambiente rico en tecnologías educativas. El docente pasa de ser el portador del conocimiento a actuar como guía de los estudiantes, facilitándoles el uso de los recursos y las herramientas que necesitan para indagar y crear nuevos conocimientos y destrezas; actúa como gestor de los recursos de aprendizaje y enfatiza su papel de orientador y mediador (Salinas, 2004). En el caso del aprendiz, este debe diseñar nuevas estrategias y rutas de aprendizaje, de llevar a cabo un aprendizaje mucho más social, más activo y, en el caso de los docentes, deben diseñar nuevas propuestas formativas que favorezcan aprendizajes más flexibles, que respondan a los nuevos retos de la sociedad y a las necesidades actuales de los estudiantes (Monsalve Gómez y Amaya Vanegas, 2014).

Garcia Aretio (2007) sostiene que rol del docente en la educación virtual debe cumplir con el objetivo de que el educando adquiera competencias valiosas para su desarrollo, es decir, que adquiera conocimientos, habilidades y actitudes, así como también que aprenda a adquirirlas por sí mismo. En este sentido, el rol del educador como orientador supone poseer ciertas competencias que permitan cumplir con las funciones del proceso educativo (García Aretio, 2007). Para un estudio anterior del ICF, se elaboró en base a los planteos de García Aretio (2007) una síntesis del mapa funcional definido por el autor que también orienta los fundamentos pedagógicos de esta propuesta (Correa, Moreira y Ureta, 2017).

Tabla 1

Funciones y competencias del rol docente en ambientes tecnológicos según García Aretio (2007)

\section{Funciones}

\section{Competencias}




\begin{tabular}{|c|c|}
\hline Académica & $\begin{array}{l}\text { Detectar necesidades de formación para distintos } \\
\text { colectivos } \\
\text { Establecer los objetivos de aprendizaje en cada acción } \\
\text { formativa } \\
\text { Determinar contenidos de acuerdo a los objetivos } \\
\text { Planificar la acción formativa a distancia en } \\
\text { colaboración con } \\
\text { otros expertos } \\
\text { Determinar la metodología a distancia adecuada para } \\
\text { cada acción } \\
\text { formativa } \\
\text { Asesorar y gestionar el ambiente de aprendizaje } \\
\text { Colaborar en la concreción, selección y diseño de } \\
\text { recursos }\end{array}$ \\
\hline Orientadora & $\begin{array}{l}\text { Motivar y facilitar el aprendizaje } \\
\text { Dinamizar el grupo y fomentar la participación } \\
\text { Favorecer la interacción y el trabajo colaborativo } \\
\text { Aclarar y resolver dudas, problemas, etc. } \\
\text { Aplicar criterios de valuación } \\
\text { Aportar la retroalimentación necesaria de cada una de } \\
\text { las fases de } \\
\text { Aprendizaje }\end{array}$ \\
\hline Gestora & $\begin{array}{l}\text { Saber utilizar las principales herramientas ofimáticas y } \\
\text { de internet } \\
\text { Conocer las características básicas de los equipos y las } \\
\text { infraestructuras informáticas } \\
\text { Saber aprovechar las posibilidades de comunicación } \\
\text { que ofrece } \\
\text { internet } \\
\text { Elaborar las guías didácticas que faciliten el proceso } \\
\text { de } \\
\text { aprendizaje } \\
\text { Proponer y elaborar los recursos necesarios } \\
\text { Conocer el funcionamiento de la institución formativa }\end{array}$ \\
\hline Evaluadora & $\begin{array}{l}\text { Precisar los momentos adecuados para la evaluación } \\
\text { continua } \\
\text { Determinar los recursos necesarios para la evaluación } \\
\text { Diseñar pruebas de evaluación de acuerdo a los } \\
\text { objetivos de }\end{array}$ \\
\hline
\end{tabular}




\begin{tabular}{|l|l|}
\hline & $\begin{array}{l}\text { aprendizaje. } \\
\text { Determinar indicadores de evaluación y de calidad }\end{array}$ \\
\hline Investigadora & $\begin{array}{l}\text { Saber acceder y utilizar las fuentes documentales } \\
\text { Saber aplicar diferentes técnicas de investigación } \\
\text { Saber elaborar los instrumentos necesarios para la } \\
\text { Investigación }\end{array}$ \\
\hline
\end{tabular}

Fuente: Correa, Moreira y Ureta (2017)

A todo lo antedicho, hay que agregar que para Maggio (2012) existen cuatro orientaciones que actualmente ofrecen los ambientes tecnológicos para las prácticas educativas: la reinterpretación curricular, los trabajos conjuntos y articulados que permiten la producción original y creativa de manera colaborativa, la producción colectiva y la evaluación de manera natural, coherente y consistente con la innovación y con el cambio que se quiere promover.

Respecto al aprendizaje autónomo en línea se pueden encontrar numerosas referencias relacionadas al concepto y otros conceptos que son vinculantes entre sí, como el autoaprendizaje, aprendizaje autodirigido o aprendizaje auto-administrado. La terminología fue variando a lo largo de los años y adquiriendo mejor precisión a partir de la investigación, la evaluación en el área y el desarrollo de tecnología para la educación a distancia, principal modalidad que utiliza este tipo de metodologías.

En la actualidad, se demanda a los seres humanos, en general, el uso de estrategias cognitivas que les permitan continuar aprendiendo toda la vida y también estrategias de autorregulación de su propia conducta, que los haga ser sujetos más eficientes en este mundo tan cambiante y lleno de transformaciones (Costa Román y García Gaitero, 2017). Para Zimmerman (2000, 2001 y 2002), Schunk y Zimmerman (2003).

El constructo de aprendizaje autorregulado puede definirse como el proceso en el que los estudiantes activan y sostienen cogniciones, conductas y afectos, los cuales son orientados hacia el logro de sus metas, o como el proceso a través del cual las actividades dirigidas por metas son instigadas y sostenidas, produciéndose todo ello de forma cíclica (Zimmerman, 2000 como se citó en García Gaitero, 2016, p.16).

La realidad obliga a aprender de forma activa. Las exigencias de formación que plantea nuestra sociedad del conocimiento y lo complejo de estos aprendizajes obligan a que toda persona tenga que decidir y controlar en cada momento cómo se puede situar en su mundo tan cambiante. Hoy, las múltiples oportunidades que las TIC ofrecen exigen un aprendiz activo que sea capaz de marcarse objetivos, seleccionar estrategias e información relevante para alcanzar los mismos y navegar entre la infinidad de información que hoy nos transversaliza (Monereo et al, 2009). 
Correa y Moreira - Experiencias en la modalidad de aprendizaje autónomo en línea para la capacita...

En definitiva, se llama autorregulación al conjunto de procesos que manejan las acciones, sentimientos y pensamientos que se ponen en marcha al realizar una actividad.

Peñalosa y Landa (2008) señalan que las estrategias de aprendizaje autorregulado se clasifican en: estrategias de ensayo, que permiten seleccionar y codificar información; estrategias de elaboración, que se usan para hacer significativa la información; y estrategias organizativas, que permiten realizar conexiones entre piezas de información.

Por otra parte, diversos autores (Madera, 2000; Hernández, 2006; Ibarra Saiz y Rodríguez Gómez, 2011; Llatás Altamirano; 2016) sostienen que, para favorecer el aprendizaje autónomo en línea o aprendizaje autodirigido en línea, son importantes algunas características personales como la autoestima, la satisfacción, la creatividad, la autodisciplina, la curiosidad y la capacidad de hacer frente a las adversidades. En estas formas de aprender, el sujeto adulto se responsabiliza de su propio aprendizaje, siendo necesarios aspectos intrínsecos del individuo como la iniciativa y la responsabilidad.

Desde otra perspectiva, Monereo y Castelló (2004) señalan que la autonomía en el aprendizaje en línea le permite al estudiante tomar decisiones que le conduzcan a regular su propio aprendizaje en función a una determinada meta y a un contexto o condiciones específicas de aprendizaje.

En la base de la definición de autonomía se halla la posibilidad del estudiante de aprender a aprender, que resulta de ser cada vez más consciente de su proceso de cognición, es decir, de la metacognición. La metacognición es un proceso que se refiere al conocimiento o conciencia que tiene la persona de sus propios procesos mentales (sobre cómo aprende) y al control del dominio cognitivo (sobre su forma de aprender) (Monereo y Barberá, 2000 como se citó en Manrique Villavicencio, 2004, p. 3).

Prieto Castillo y Van de Pol (2006) plantean que actualmente existen dos grandes paradigmas o enfoques sobre el aprendizaje autónomo en línea: uno de ellos se centra en las tecnologías, haciendo énfasis en las herramientas disponibles y que tiende a ver el aprendizaje como el resultado del uso de tales tecnologías; mientras que el otro se centra en lo pedagógico, en la experiencia dialógica entre los actores del proceso de aprendizaje. En este sentido, el aprendizaje autónomo en línea es un sistema que beneficia el desarrollo integral del estudiante, asegurando un aprendizaje permanente. Dadas sus características y ventajas, la educación virtual es el ambiente perfecto para motivar su práctica, ya que permite aprender desde cualquier lugar, en un horario flexible y con un ritmo de aprendizaje personal. 
Finalmente, para Solórzano-Mendoza (2017) el aprendizaje autónomo

es el proceso intelectual, mediante el cual el sujeto pone en ejecución estrategias cognitivas y metacognitivas, secuenciales, objetivas, procedimentales y formalizadas para obtener conocimientos estratégicos. Este proceso está regido por principios de acción como: un interés manifiesto en razones que motiven la actuación deliberada; el reconocimiento de experiencias de aprendizaje previas; el establecimiento de nuevas relaciones entre aprendizaje - trabajo - vida cotidiana, así como entre teoría y práctica; la identificación de la motivación intrínseca y el desarrollo del potencial personal de la autorregulación (p. 245).

\section{Acciones realizadas para promover el aprendizaje autónomo en línea en las experiencias educativas}

En la siguiente tabla descriptiva, se presentan las acciones realizadas en los dos cursos que integran esta experiencia educativa, se definen objetivos de aprendizaje de los cursos y se muestran las actividades, recursos tecnológicos, objetos de aprendizaje y evaluaciones que se diseñaron para promover el aprendizaje autónomo en línea.

Descripción de las acciones realizadas para la promoción del aprendizaje autónomo en línea en los cursos

Tabla 2

Conceptos Básicos sobre Salud y Seguridad

\begin{tabular}{|c|c|c|c|c|}
\hline $\begin{array}{l}\text { Objetivos de } \\
\text { aprendizaje }\end{array}$ & $\begin{array}{l}\text { Objetos de } \\
\text { aprendizaje }\end{array}$ & $\begin{array}{c}\text { Recursos } \\
\text { tecnológicos } \\
\text { diseñados } \\
\text { para el } \\
\text { aprendizaje } \\
\text { autónomo en } \\
\text { línea }\end{array}$ & $\begin{array}{l}\text { Actividades } \\
\text { realizadas } \\
\text { para fomentar } \\
\text { el aprendizaje } \\
\text { autónomo en } \\
\text { línea }\end{array}$ & $\begin{array}{c}\text { Evaluación de } \\
\text { la propuesta }\end{array}$ \\
\hline $\begin{array}{l}\text { Introducir } \\
\text { conceptos } \\
\text { relacionados } \\
\text { con la salud y } \\
\text { la seguridad en } \\
\text { el trabajo e } \\
\text { identificar } \\
\text { conductas y/o } \\
\text { hábitos en el } \\
\text { marco de la } \\
\text { promoción de la }\end{array}$ & \begin{tabular}{|l|} 
Evaluación \\
diagnóstica a \\
partir de la \\
aplicación de \\
una encuesta \\
en Eva para \\
identificar los \\
conceptos \\
previos sobre la \\
temática del \\
curso.
\end{tabular} & $\begin{array}{l}\text { Curso } \\
\text { asincrónico } \\
\text { diseñado en la } \\
\text { plataforma EVA } \\
\text { (Moodle } 3.8 .9 \text { ), } \\
\text { distribuido el } \\
\text { temario y } \\
\text { actividades en } \\
\text { cuatro } \\
\text { semanas. }\end{array}$ & $\begin{array}{l}\text { Tutorías } \\
\text { docentes a } \\
\text { través de foros } \\
\text { específicos } \\
\text { para consultas. } \\
\text { Foros de } \\
\text { intercambio } \\
\text { entre los } \\
\text { participantes } \\
\text { para fomentar } \\
\text { la reflexión y el }\end{array}$ & $\begin{array}{l}\text { Aplicación del } \\
\text { formulario } \\
\text { institucional de } \\
\text { evaluación de } \\
\text { cursos y de } \\
\text { docentes del } \\
\text { ICF, completar } \\
\text { el mismo es un } \\
\text { requisito para la } \\
\text { certificación. }\end{array}$ \\
\hline
\end{tabular}




\begin{tabular}{|c|c|c|c|}
\hline $\begin{array}{l}\text { salud para } \\
\text { lograr un } \\
\text { ambiente de } \\
\text { trabajo más } \\
\text { saludable. } \\
\text { Conocer y } \\
\text { actuar en el } \\
\text { marco } \\
\text { normativo } \\
\text { laboral vigente } \\
\text { de la Udelar. } \\
\text { Promover la } \\
\text { reflexión grupal } \\
\text { sobre distintas } \\
\text { temáticas } \\
\text { comunes y que } \\
\text { a través de } \\
\text { intervenciones } \\
\text { se comparta y } \\
\text { enriquezca } \\
\text { sobre las } \\
\text { posibles } \\
\text { soluciones en } \\
\text { cada ambiente } \\
\text { de trabajo. }\end{array}$ & $\begin{array}{l}\text { Foro de } \\
\text { presentación de } \\
\text { los } \\
\text { participantes } \\
\text { cuyo objetivo } \\
\text { es la } \\
\text { interrelación y } \\
\text { favorecer el } \\
\text { ambiente de } \\
\text { aprendizaje. } \\
\text { Polimedias } \\
\text { informativas } \\
\text { sobre } \\
\text { conceptos } \\
\text { básicos de } \\
\text { salud y } \\
\text { seguridad en el } \\
\text { trabajo } \\
\text { elaboradas por } \\
\text { el IcF en } \\
\text { contexto de } \\
\text { pandemia. } \\
\text { Fichas de } \\
\text { lectura. } \\
\text { Animaciones } \\
\text { audiovisuales } \\
\text { disponibles en } \\
\text { la Red. }\end{array}$ & $\begin{array}{l}\text { trabajo } \\
\text { colaborativo } \\
\text { sobre las } \\
\text { distintas } \\
\text { temáticas } \\
\text { abordadas. } \\
\text { Glosario } \\
\text { colaborativo. } \\
\text { La evaluación } \\
\text { de los } \\
\text { aprendizajes } \\
\text { consistió en } \\
\text { una tarea final } \\
\text { que implicaba } \\
\text { identificar } \\
\text { alguna de las } \\
\text { temáticas } \\
\text { abordadas en } \\
\text { el curso, } \\
\text { presentes en su } \\
\text { lugar de } \\
\text { trabajo, } \\
\text { identificar a qué } \\
\text { clase de riesgo } \\
\text { se estarían } \\
\text { exponiendo y } \\
\text { cuáles podrían } \\
\text { ser las } \\
\text { consecuencias } \\
\text { si no se trata, } \\
\text { finalmente, se } \\
\text { solicitaba } \\
\text { presentar una } \\
\text { propuesta para } \\
\text { solucionar } \\
\text { dicho problema. }\end{array}$ & $\begin{array}{l}\text { Aplicación de } \\
\text { encuesta } \\
\text { elaborada por } \\
\text { el equipo } \\
\text { docente del } \\
\text { curso sobre } \\
\text { expectativas } \\
\text { relacionadas a } \\
\text { los } \\
\text { conocimientos } \\
\text { y sus posibles } \\
\text { aplicaciones en } \\
\text { el ámbito de } \\
\text { trabajo. }\end{array}$ \\
\hline
\end{tabular}

Fuente: Elaboración propia

Tabla 3

Herramientas y aplicaciones de la Web 3.0 para teletrabajar 


\begin{tabular}{|c|c|c|c|c|}
\hline $\begin{array}{l}\text { Objetivos de } \\
\text { aprendizaje }\end{array}$ & $\begin{array}{l}\text { Objetos de } \\
\text { aprendizaje }\end{array}$ & $\begin{array}{c}\text { Recursos } \\
\text { tecnológicos } \\
\text { diseñados } \\
\text { para el } \\
\text { aprendizaje } \\
\text { autónomo en } \\
\text { línea }\end{array}$ & $\begin{array}{c}\text { Actividades } \\
\text { realizadas } \\
\text { para fomentar } \\
\text { el aprendizaje } \\
\text { autónomo en } \\
\text { línea }\end{array}$ & $\begin{array}{l}\text { Evaluación de } \\
\text { la propuesta }\end{array}$ \\
\hline $\begin{array}{l}\text { Adquirir } \\
\text { conocimientos } \\
\text { sobre } \\
\text { herramientas y } \\
\text { aplicaciones } \\
\text { informáticas } \\
\text { para la mejora } \\
\text { del desempeño } \\
\text { laboral. } \\
\text { Aplicar las } \\
\text { herramientas y } \\
\text { aplicaciones a } \\
\text { las diferentes } \\
\text { actividades } \\
\text { laborales: } \\
\text { comunicación, } \\
\text { trabajo en } \\
\text { equipo, trabajo } \\
\text { colaborativo, } \\
\text { organización de } \\
\text { tareas. }\end{array}$ & \begin{tabular}{|l} 
Aplicación de \\
un cuestionario \\
en EvA sobre la \\
experiencia de \\
teletrabajo y del \\
aprendizaje \\
autónomo en \\
línea. \\
Video \\
introductorio \\
sobre las \\
temáticas a \\
tratar en cada \\
módulo. \\
Polimedias \\
informativas \\
sobre \\
herramientas \\
de la Web y \\
tutoriales \\
aplicativos. \\
Fichas de \\
lectura. \\
Simuladores de \\
aplicativos. \\
Lista de dudas \\
y preguntas \\
frecuentes \\
respecto al \\
módulo para \\
fomentar la \\
autonomía de \\
participantes.
\end{tabular} & $\begin{array}{l}\text { Curso } \\
\text { asincrónico } \\
\text { diseñado en la } \\
\text { plataforma EVA } \\
\text { (Moodle } 3.8 .9 \text { ), } \\
\text { distribuido el } \\
\text { temario y } \\
\text { actividades en } \\
\text { seis solapas, } \\
\text { con una } \\
\text { duración de } \\
\text { tres semanas. }\end{array}$ & $\begin{array}{l}\text { Tutorías } \\
\text { docentes a } \\
\text { través de foros } \\
\text { específicos } \\
\text { para consultas. } \\
\text { Actividades } \\
\text { sincrónicas a } \\
\text { través del uso } \\
\text { de la } \\
\text { plataforma } \\
\text { Zoom. } \\
\text { foros de } \\
\text { intercambio } \\
\text { entre los } \\
\text { participantes } \\
\text { para fomentar } \\
\text { la reflexión y el } \\
\text { trabajo } \\
\text { colaborativo } \\
\text { sobre las } \\
\text { distintas } \\
\text { temáticas } \\
\text { abordadas. } \\
\text { Glosario } \\
\text { colaborativo. } \\
\text { Prácticas } \\
\text { específicas con } \\
\text { aplicaciones en } \\
\text { situaciones } \\
\text { para el trabajo } \\
\text { como por } \\
\text { ejemplo un } \\
\text { diseño en } \\
\text { Canva, un }\end{array}$ & \begin{tabular}{|l|} 
Aplicación del \\
formulario \\
institucional de \\
evaluación de \\
cursos y de \\
docentes del \\
ICF, completar \\
el mismo es un \\
requisito para la \\
certificación. \\
Aplicación de \\
un cuestionario \\
elaborado por \\
el equipo \\
docente que \\
valoraba la \\
experiencia de \\
aprendizaje \\
autónomo en \\
línea. \\
Una instancia \\
de \\
autoevaluación \\
por parte de los \\
participantes a \\
través de la \\
plataforma \\
Zoom.
\end{tabular} \\
\hline
\end{tabular}




\begin{tabular}{|l|l|l|}
\hline & & documento \\
colaborativo en & \\
Google Drive o \\
un proyecto en \\
Monday.com \\
La evaluación \\
de los \\
aprendizajes \\
consistió en un \\
mínimo de \\
tareas \\
realizadas en \\
cada módulo. \\
Lista de \\
chequeo de las \\
actividades y \\
lectura \\
recomendadas \\
al cierre de \\
cada módulo
\end{tabular} \mid

Fuente: Elaboración propia

\section{Resultados}

Se recibieron 318 solicitudes de inscripción a los cursos bajo esta modalidad de aprendizaje autónomo en línea, siendo los que culminaron el proceso y las exigencias de aprobación un poco más de la mitad de los inscriptos (50,62 \%). El restante $49,38 \%$ representan a los participantes que se inscribieron y no cursaron, comenzaron los cursos, pero no lograron culminar las tareas solicitadas o abandonaron el curso durante el proceso de desarrollo del curso. En la siguiente tabla se detallan inscripciones y aprobaciones por cada curso.

Tabla 4

Cantidad de inscripciones y aprobaciones por curso bajo la modalidad de aprendizaje autónomo

\begin{tabular}{|l|l|l|l|}
\hline \multicolumn{1}{|c|}{ Curso } & \multicolumn{1}{c|}{ Inscriptos } & \multicolumn{1}{c|}{ Aprobaron } & \% de aprobación \\
\hline $\begin{array}{l}\text { Conceptos Básicos } \\
\text { sobre Salud y } \\
\text { Seguridad }\end{array}$ & 105 & 56 & $53,33 \%$ \\
\hline $\begin{array}{l}\text { Herramientas y } \\
\text { aplicaciones de la }\end{array}$ & 213 & 105 & $49,29 \%$ \\
\hline
\end{tabular}




\begin{tabular}{|l|l|l|l|}
\hline $\begin{array}{l}\text { Web 3.0 para } \\
\text { teletrabajar }\end{array}$ & & & \\
\hline Total & 318 & 161 & $50,62 \%$ \\
\hline
\end{tabular}

Fuente: Elaboración propia en base a datos aportados por bedelía

Para evaluar las experiencias, se implementó un cuestionario al inicio del curso y otro al finalizar el proceso de enseñanza-aprendizaje, en el primer cuestionario, se solicitó a todos los participantes (161 participantes activos) responder las siguientes preguntas: ¿Qué entiende por aprendizaje autónomo en línea? ¿Ha realizado cursos en la modalidad de aprendizaje autónomo en línea? y ¿Cuáles son sus expectativas respecto del curso y la propuesta de aprendizaje?

En esta primera consulta sobre «¿Qué entiende por aprendizaje autónomo en línea?» se destacan las siguientes respuestas que tienen relación con el marco teórico anteriormente expuesto, sobre todo con aquellos aspectos relacionados a las conductas, disciplinamiento, regulación del tiempo y administración de horarios:

El aprendizaje autónomo en línea es una forma de aprender, debemos tener los medios a nuestro alcance para lograrlo. Lo entiendo como la capacidad que tenemos los individuos de desarrollar nuevos conocimientos a través de la experimentación, la observación y el estudio de diferentes situaciones o temáticas con las cuales entramos en contacto en diversos contextos (Mujer, escalafón servicios generalesvigilancia).

Se trata de un proceso de adquisición de conocimientos, habilidades, valores y actitudes, que la persona realiza por su cuenta ya sea mediante el estudio o la experiencia. Una modalidad de formación en la cual uno mismo ejecuta, planifica y controla los contenidos a estudiar (Varón, escalafón administrativo).

Mayormente, los participantes relacionan el aprendizaje autónomo en línea a las siguientes características: la autorregulación, la administración del tiempo y la regulación del cumplimiento de las diferentes actividades que implica aprobar el curso. En menor proporción se destacan la posibilidad que ofrece la asincronía, el ensayo y el error, el diseño de los materiales de estudio, la tutoría docente y la motivación del participante para adquirir nuevos conocimientos.

La segunda dimensión consultada en el primer cuestionario da cuenta de que menos de la mitad de los participantes $(41,67 \%)$ ya habían realizado previamente cursos en la modalidad de aprendizaje autónomo en línea, implicando que el 58,33 \% accedía por primera vez a este tipo de formaciones en línea, siendo un desafío extra para los tutores, teniendo este dato significativo sobre el perfil de los participantes que tendía a formarse bajo otras modalidades de aprendizaje más tradicionales. 
Por último, se solicitó que expresaran cuáles eran las expectativas que tenían respecto del curso y la propuesta de aprendizaje, destacándose las siguientes respuestas:

Aprender herramientas que me ayuden a realizar el teletrabajo y a manejar recursos informáticos que me permitan trabajar en equipo a la distancia. Ampliar conocimientos en el manejo de las aplicaciones y herramientas con las que ya se trabaja, incorporar manejo de nuevas herramientas y/o aplicaciones, así como utilidades facilitadoras dentro de éstas (Varón, escalafón administrativo).

Espero adquirir habilidades para mejorar o efectivizar las tareas, hasta ahora creo que determinadas tareas a distancia son muy difíciles o imposibles de realizar, y/o minimizar sus dificultades (Mujer, escalafón servicios generales-vigilancia).

En este ítem se observa nuevamente que la motivación principal es aplicar los conocimientos adquiridos al ámbito laboral y la motivación personal, que estuvo marcada por el contexto de la pandemia, dado que los contenidos abordados en los cursos referían a la salud, a la seguridad y a brindar herramientas para el teletrabajo.

Al finalizar los cursos, se solicitó nuevamente a los participantes que completen un nuevo cuestionario, en este caso, referida a la evaluación de la modalidad de aprendizaje autónomo en línea. Uno de los primeros ítems del cuestionario apuntaba a conocer si el tiempo sugerido para la realización del curso era adecuado o no. El $55,5 \%$ de los participantes percibió que el tiempo sugerido para completar el curso era adecuado o muy adecuado, mientras que un $44,5 \%$ percibió como poco adecuado o nada adecuado el tiempo establecido. En el gráfico siguiente se presentan los porcentajes obtenidos sobre la percepción, en relación a la pertinencia en el tiempo de duración del curso. 


\section{Gráfico 1}

Percepción del tiempo de duración del curso en modalidad de aprendizaje autónomo en línea

\section{Tiempo de duración del curso}

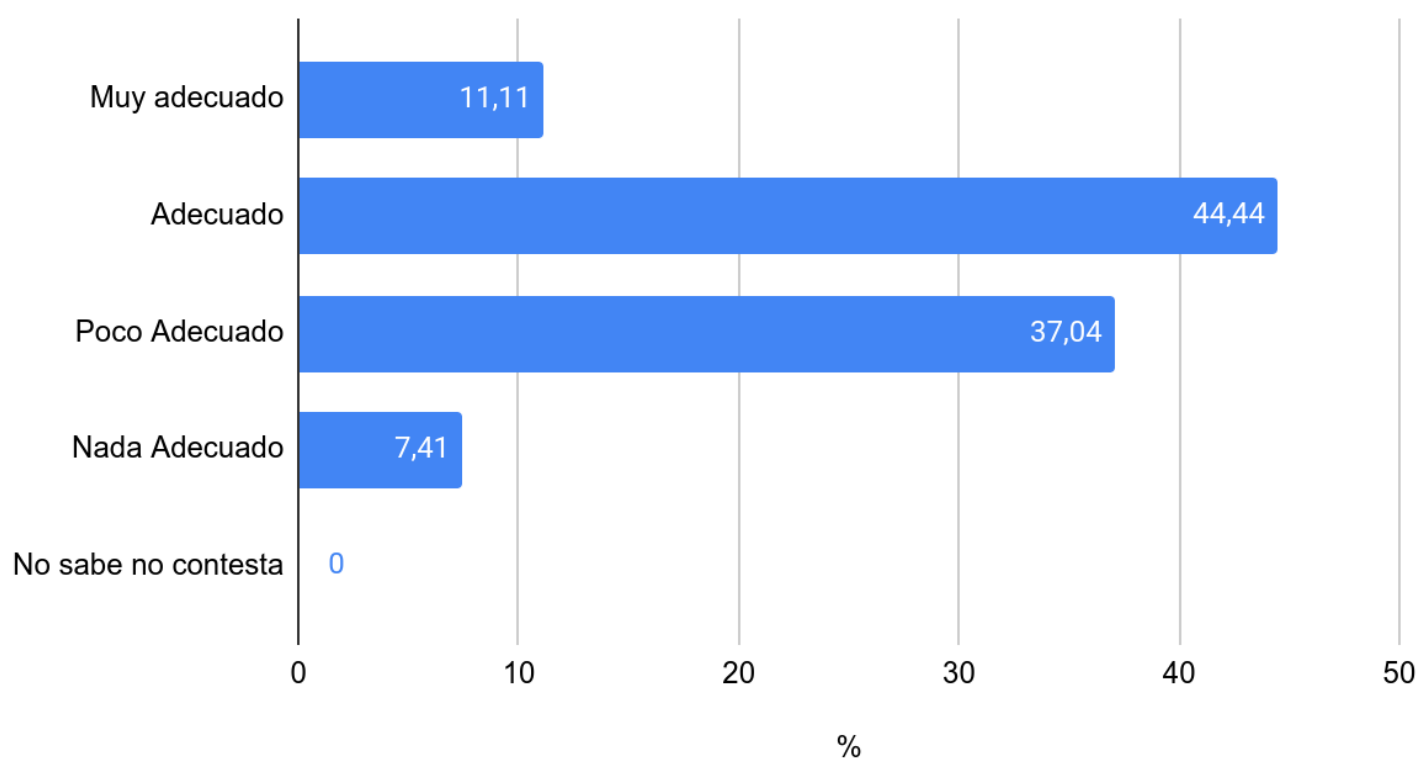

Fuente: Elaboración propia

Se infiere que estos resultados pueden relacionarse con la administración personal de los tiempos, la sobrecarga de trabajo que implicó el cambio a la modalidad de teletrabajo (Venegas Tresierra y Leyva Pozo, 2020) y, debido a que el $65 \%$ de quienes participaron fueron mujeres, se puede suponer que se relacione el dato con la conciliación de la vida profesional y la vida familiar.

Quienes presentaron alguna dificultad para completar el curso, entre los principales motivos que expresaron, se encuentran: que les insumió más tiempo de lo previsto la realización de las diferentes actividades $(29,63 \%)$, mientras que en igual porcentaje $(11,11 \%)$ se encuentran los motivos personales y la organización del tiempo; como se puede observar en la tabla 5 , si bien no fue consultado cuáles fueron los motivos personales que dificultaron el avance del curso, se puede inferir, como se mencionó anteriormente, que puede relacionarse con el perfil feminizado de la población que realizó el curso.

\section{Tabla 5}

Principales motivos que interfirieron en el avance del curso en el tiempo estipulado al inicio - valores expresados en porcentajes

\begin{tabular}{|c|c|}
\hline Motivos que dificultaron el avance en el curso & \multicolumn{1}{c|}{$\%$} \\
\hline No tuve dificultades con el tiempo establecido & 62,96 \\
\hline
\end{tabular}




\begin{tabular}{|l|l|}
\hline $\begin{array}{l}\text { Motivos personales que dificultaron el avance y } \\
\text { seguimiento del curso }\end{array}$ & 11,11 \\
\hline $\begin{array}{l}\text { Me llevó más tiempo de lo previsto las lecturas y } \\
\text { ejercicios }\end{array}$ & 29,63 \\
\hline Me costó organizar los tiempos de avance y seguimiento & 11,11 \\
\hline $\begin{array}{l}\text { Me costó ser disciplinado/a para el aprendizaje autónomo } \\
\text { en línea }\end{array}$ & 7,41 \\
\hline Otras situaciones que se presentaron & 3,7 \\
\hline
\end{tabular}

Fuente: Elaboración propia

Si se observa el $29,63 \%$, probablemente se encuentren diversos factores incidiendo en esta situación que den respuesta al porqué de esta dificultad y que pueden relacionarse a la escasa experiencia de los participantes en esta modalidad de aprendizaje autónomo en línea, que también puede reflejarse en los porcentajes minoritarios como en "Me costó organizar los tiempos de avance y seguimiento» $(11,11 \%)$ y «Me costó ser disciplinado para el aprendizaje autónomo en línea» $(7,41$ $\%)$, esto pudo deberse a las habilidades que se necesitaban para avanzar en el curso y a una no adecuada distribución y organización personal para avanzar y completar las actividades.

Este segundo cuestionario indagó también si se consideraba que las tutorías recibidas eran suficientes para avanzar en el curso y si en esta modalidad era necesario contar con tutorías docentes para cursar en los tiempos establecidos, encontrando que el $81,48 \%$ de los participantes observaron como suficientes y convenientes las tutorías en las propuestas de aprendizaje autónomo en línea y un 62,96 \% manifestó que eran necesarias o muy necesarias contar con tutorías docentes en este tipo de modalidad. Asimismo, el 77,78 \% de los participantes que cursaron «Herramientas y aplicaciones de la Web 3.0 para teletrabajar» afirmaron que las sesiones en vivo permitieron un mejor desempeño en el curso, mientras que el $85,19 \%$ de los participantes que cursaron "Conceptos Básicos de Salud y Seguridad», que no contaron con sesiones en vivo, pero sí con foros que permitían acceder a tutorías y aclarar dudas, afirmaron que esta herramienta de comunicación fue imprescindible para despejar dudas y avanzar en el curso.

Finalmente, se solicitó que se expresara una valoración global de las experiencias percibidas por los participantes sobre la modalidad de aprendizaje autónomo en línea, en donde se destacan, a continuación, aquellas respuestas recibidas que presentan mayor conformidad con la modalidad y con los objetivos planteados al momento del diseño de los cursos: 
El curso es excelente: Volumen de contenido super completo con materiales muy bien seleccionados (a veces era difícil mirar todo, porque era bastante, pero es cuestión de organizarse, seguramente voy a volver a revisar los módulos de nuevo). Los videos introduciendo cada módulo estaban muy claros y era como tener la clase en vivo y en directo y las tareas eran acordes a los contenidos, no eran tan complicadas, salvo algún tema específico que tenía un poco más de nivel de complejidad. Un módulo en especial me hubiera gustado trabajarlo en una clase virtual (sincrónica) para conocer las experiencias de otros participantes, evacuar dudas y conocer las opiniones de las docentes al respecto de cada propuesta (Varón, escalafón administrativo).

Fue acertada la herramienta Zoom para dictarlos. Y serían mejor en horarios que NO coincidan con los horarios de trabajo de algunos funcionarios, generando grupos con un mejor intercambio entre docentes y alumnos, en un ambiente más tranquilo y con menos distracciones. (Varón, escalafón servicios generales - vigilancia).

El curso fue súper enriquecedor, interesante, adquirí muchos conocimientos nuevos y aplicación de técnicas y herramientas que fueron muy prácticas y aplicables al trabajo que desempeño en la Udelar y en mi vida personal. Me resultó exigente en tiempo, por el tiempo que yo dispongo y porque había mucha información, herramientas y conceptos nuevos para aprender; así mismo fue accesible, ameno, interesante y motivador adquirir nuevos conocimientos y aprender a aplicarlos, para así realizar las tareas en forma más eficiente, facilitando el trabajo y la comunicación del equipo de trabajo y pudiendo optimizar el trabajo con un montón de herramientas que uno sabía que existían pero no sabía usarlas o como aplicarlas al trabajo. Considero que este curso me aportó muchos conocimientos y me generó motivación para implementar formas nuevas de desarrollar las tareas con mi equipo de trabajo (Mujer, escalafón administrativo).

Como sugerencia es que quien dicte la clase debe tener la capacidad para no cortarla en cada momento $\mathrm{x}$ comentarios de situaciones puntuales que manifiestan los compañeros, para eso está el foro en EVA (Mujer, escalafón administrativo)».

\section{Conclusiones}

A partir de estas experiencias se considera que el ICF debe contar en su oferta con cursos en modalidad de aprendizaje autónomo en línea, debido a que existe demanda por parte del funcionariado y permite un mayor alcance, que se demuestra en el número de inscriptos y en el número de aprobados.

La modalidad de aprendizaje autónomo en línea exige acompañar y hacer un seguimiento de este, como se observa en el rol preponderante que ejercieron las tutorías en estas dos propuestas, para que se mantenga la comunicación, la motivación, la flexibilidad de tiempos cuando fue requerida por los participantes y tal como fuera planteado anteriormente en Salinas (2004) y García Aretio (2007). 
Se debe agregar que estas propuestas se generaron en respuesta al contexto de pandemia que requirió de nuevas habilidades de funcionariado para organizarse, lograr el trabajo colaborativo, comunicarse y dar respuesta a los nuevos retos y necesidades actuales de la modalidad de teletrabajo, en este sentido, la intencionalidad de ofrecer cursos de aprendizaje autónomo en línea se originó para contar con mayor disponibilidad del funcionariado para elegir esta modalidad de aprendizaje.

A decir de Maggio (2012), los cursos que se ofrecen en esta modalidad, bajo ambientes tecnológicos enriquecidos, deben ser rediseñados y reconfigurados, evitando la mera reproducción de las experiencias presenciales. En este sentido, el curso de "Herramientas y aplicaciones de la Web 3.0 para teletrabajar» se diseña específicamente para atender una demanda constatada a partir del confinamiento, mientras que en curso de «Salud y Seguridad» fue rediseñado y adaptado al contexto de la pandemia.

Respecto a las propuestas de evaluación de los cursos, buscaron la aplicación de herramientas en el ambiente de trabajo, pero también la producción original y creativa del participante para resolver situaciones laborales, así como la colaboración entre el funcionariado para el logro de los aprendizajes y la experimentación del trabajo colaborativo.

Se identifica, en ambas experiencias, lo planteado por Peñalosa y Landa (2008) cuando se promovió el desarrollo de estrategias de ensayo, que permitiera seleccionar y codificar información, estrategias de elaboración, que se usaron para hacer significativa la información y estrategias organizativas, que permiten realizar conexiones entre piezas de información. Cabe destacar que la producción realizada por los participantes en forma colaborativa originó una guía disponible para ser utilizada en posteriores cursos y para colaborar en la actividad cotidiana de las personas trabajadoras que posteriormente fue publicada en el repositorio institucional del ICF.

A través de la percepción de los participantes de los cursos, se evidencia principalmente como mayor dificultad la organización y la regulación del tiempo para culminarlos, constando algunas características del aprendizaje autónomo en línea que se identifican con las debilidades de esta modalidad: desvinculación, abandono, dificultad para organizar los tiempos, entre otros. Esta situación generó que el ICF diseñara, a posteriori, una nueva propuesta de capacitación cuyo objetivo fue conocer y ejercitar herramientas para administrar el tiempo y la organización del trabajo en modalidad remoto. 
Los resultados dan cuenta que el funcionariado, en su mayoría, no ha participado de esta modalidad, ni en el ICF ni en otras instituciones educativas, es por ello que resulta imprescindible que la oferta de cursos con esta modalidad de aprendizaje, sea lo suficientemente amplia, diversa, adaptada a las necesidades y demandas de capacitación y sostenida a través del tiempo, para que el funcionariado tenga disponible en la oferta esta forma de aprender, así como otras modalidades de aprendizaje.

Por último, el contexto de pandemia facilitó un mayor desarrollo de propuestas de este estilo y también una preferencia entre los usuarios para este tipo de modalidades, debido a que sus características eran las más adecuadas para el contexto de confinamiento. Sin embargo, es fundamental incluir de manera sostenida esta modalidad para la gestión del conocimiento del funcionariado técnico, administrativo y de servicios de la Udelar.

\section{Referencias Bibliográficas}

Argüelles Pabón, D. (2010). Estrategias para promover procesos de aprendizaje autónomo. Revista Escuela de Administración de Negocios, (69), 150. Recuperado de: https://journal.universidadean.edu.co/index.php/Revista/article/view/528

C.D.C. (2018). Ordenanza del Instituto de Capacitación y Formación «José Jorge (Tito) Martínez Fontana» de los Funcionarios Técnicos, Administrativos y de Servicio. Res. No 17 de C.D.C. de 15/V/2018 - Dist. 343/18 - D.O. 17/VIII/2018. Recuperado de: https://dgjuridica.udelar.edu.uy/wp-content/uploads/2016/05/Ordenanza-312.pdf

Correa, A. Moreira, L. y Ureta, X. (2017). La formación e-learning frente a la formación presencial en personas adultas trabajadoras. En Dubini, L. (Presidente). Actas del IX Congreso Iberoamericano de Educación Científica y del I Seminario de Inclusión Educativa y Sociodigital (CIEDUC 2017). Calidad de los procesos formativos de los Docentes de Ciencias. Integración curricular de las TIC a la enseñanza y aprendizaje.[Presentación oral]. ISBN 978-84-16978-21-2. Mendoza, Argentina.

Costa Román, S., \& García Gaitero, S. (2017). El aprendizaje autorregulado y las estrategias de aprendizaje / SELF REGULATED LEARNING AND LEARNING STRATEGIES. Tendencias pedagógicas, 30(2017). https://doi.org/10.15366/tp2017.30.007

García Aretio, L. (Coord.) (2007). De la educación a distancia a la educación virtual. Barcelona: Ariel, 303. ISBN: 978-84-344-2665-5.

García Gaitero, O. (2016). El aprendizaje autorregulado en la enseñanza del inglés para la mejora de la producción escrita en el tercer ciclo de la educación primaria en la enseñanza bilingüe en la Comunidad de Madrid [Tesis de Doctorado, 
Universidad Complutense de Madrid]. Repositorio Institucional - Universidad Complutense de Madrid.

Hernández, G. (2006), Miradas constructivistas en psicología de la educación. México: Paidós.

Ibarra Saiz M. y Rodríguez Gómez, G. (2011). Aprendizaje autónomo y trabajo en equipo: reflexiones desde la competencia percibida por los estudiantes universitarios. Revista Electrónica Interuniversitaria de Formación del Profesorado, 14(4), 73-85. Disponible en: http://www.redalyc.org/pdf/2170/217022117006.pdf

Llatas Altamirano, L. (2016). Programa Educativo para el Aprendizaje Autónomo basado en Estrategias didácticas fundamentadas en el uso de las tecnologías y la comunicación. La investigación formativa de los estudiantes del primer ciclo de la USAT (Tesis doctoral). Universidad Católica Santo Toribio de Mogrovejo (USAT), Chiclayo, Perú.

Madera, I. (2000). Internacionalización, calidad y formación continua en la universidad latinoamericana: desafíos para su desarrollo en el entorno global: Caso República Dominicana. En Fröhlich, W. y Jütte, W. (Eds.), Universidad y educación continua. Nuevos desarrollos en Latinoamérica y Europa. Austria: Donau- Universität Krems.

Maggio, M. (2012). Enriquecer la enseñanza. Los ambientes con alta disposición tecnológica como oportunidad. Buenos Aires: Paidós

Manrique Villavicencio, L. (2004, marzo 23-abril 4). El aprendizaje autónomo en la educación a distancia [Presentación oral]. Primer Congreso virtual latinoamericano de educación a distancia, Buenos Aires, Argentina, 1-11. https://seminario-taller-apa-micea-tic.webnode.com.ar/_files/200000014-3bf4e3ce fb/APRENDIZAJE_AUTONOMO_A_DISTANCIA.pdf,

Monereo, C. y Barberá, E. (2000). Diseño instruccional de las estrategias de aprendizaje en entornos educativos no-formales. En Monereo et al. Estrategias de aprendizaje. Madrid: Ediciones de la Universitat Oberta de Catalunya.

y Castelló, M. (2004). Un modelo para el análisis de contextos de asesoramiento psicopedagógico en educación formal. En Badia, A., Mauri, T. y Monereo, C. (Coords.). La práctica psicopedagógica en educación formal. Barcelona: UOC.

y Otros (2009). Ser un docente estratégico: cuando cambiar la estrategia no basta. En Cultura y Educación, 21(3).

Monsalve Gómez, J. C. y Amaya Vanegas, D. M. (2014). Implementación de ambientes de aprendizaje b-learning: retos para docentes y estudiantes. Revista Colombiana de Ciencias Sociales, 5(2), 408-417.

Peñalosa, E. y Landa, P. (2008). Objetos de aprendizaje: una propuesta de conceptualización, taxonomía y metodología. Revista Electrónica de Psicología Iztacala, 11 (3), (19-49). [en línea] Disponible en: http://www. iztacala.unam.mx/carreras/psicologia/ psiclin/principal.html 
Prieto Castillo, D. y van de Pol, P. (2006). E-learning, comunicación y educación: el diálogo continúa en el ciberespacio. Bogotá: RNTC.

Salinas, J. (2004). Innovación docente y uso de las TIC en la enseñanza universitaria. RUSC. Revista Universidades y Sociedad del Conocimiento, 1 (1), 1-16. [Fecha de Consulta 2 de Julio de 2021]. ISSN:. Disponible en: https://www.redalyc.org/articulo.oa?id=78011256001

Schunk, D.H. y Zimmerman, B.J. (2003). Autorregulación y aprendizaje. En Reynolds, W. y Miller, G. (Eds.), Manual de psicología: psicología educativa, vol. 7. USA: John Wiley \& Sons Inc, págs. 59-78.

Solórzano-Mendoza, Y. D. (2017). Aprendizaje autónomo y competencias. Dominio de las Ciencias, 3, 241-253. https://doi.org/10.23857/dc.v3i1.390.

Venegas Tresierra C., Leyva Pozo A. (2020). La fatiga y la carga mental en los teletrabajadores: a propósito del distanciamiento social. Revista Especializada en Salud Pública. 94 (1). Recuperado de: https://www.mscbs.gob.es/biblioPublic/publicaciones/recursos_propios/resp/revist a_cdrom/VOL94/REVISIONES/RS94C_202010112.pdf

Zimmerman, B.J. (2000). Attaining self-regulation: A social cognitive perspective. En M. Boekaerts, P. R. Pintrich y M. Zeidner (Eds.) Handbook of self-regulation. San Diego, CA: Academic Press, 451- 502.

(2001). Achieving academic excellence: A self-regulatory perspective. En M.

Ferrari (Ed.) The pursuit of excellence through education. Mahwah, NJ: Erlbaum, 85-110.

(2002). Becoming self-regulated learned: An overview. En Theory into Practice, 41(1), 64-72. 